PROCEEDINGS OF THE

AMERICAN MATHEMATICAL SOCIETY

Volume 136, Number 11, November 2008, Pages 3883-3893

S 0002-9939(08)09537-3

Article electronically published on June 24, 2008

\title{
CONVERGENT MARTINGALES OF OPERATORS AND THE RADON NIKODÝM PROPERTY IN BANACH SPACES
}

\author{
STUART F. CULLENDER AND COENRAAD C. A. LABUSCHAGNE
}

(Communicated by N. Tomczak-Jaegermann)

\begin{abstract}
We extend Troitsky's ideas on measure-free martingales on Banach lattices to martingales of operators acting between a Banach lattice and a Banach space. We prove that each norm bounded martingale of cone absolutely summing (c.a.s.) operators (also known as 1-concave operators), from a Banach lattice $E$ to a Banach space $Y$, can be generated by a single c.a.s. operator. As a consequence, we obtain a characterization of Banach spaces with the Radon Nikodým property in terms of convergence of norm bounded martingales defined on the Chaney-Schaefer $l$-tensor product $E \widetilde{\otimes}_{l} Y$. This extends a classical martingale characterization of the Radon Nikodým property, formulated in the Lebesgue-Bochner spaces $L^{p}(\mu, Y)(1<p<\infty)$.
\end{abstract}

\section{INTRODUCTION}

Let $(\Omega, \Sigma, \mu)$ be a finite measure space and let $Y$ be a Banach space. For $1 \leq$ $p<\infty$, let $L^{p}(\mu, Y)$ denote the space of (classes of a.e. equal) Bochner $p$-integrable functions $f: \Omega \rightarrow Y$ and denote the Bochner norm on $L^{p}(\mu, Y)$ by $\Delta_{p}$, i.e.

$$
\Delta_{p}(f)=\left(\int_{\Omega}\|f\|_{Y}^{p} d \mu\right)^{1 / p} .
$$

In the Lebesgue-Bochner spaces, martingale theory provides an important link to the geometric properties of the Banach space $Y$. For example, $Y$ has the Radon Nikodým property if and only if every martingale $\left(f_{i}\right) \subset L^{p}(\mu, Y)$, which is uniformly $\|\cdot\|_{p}$-bounded, converges in the $\|\cdot\|_{p}$-norm for all finite measure spaces $(\Omega, \Sigma, \mu)$ and $1<p<\infty$ (cf. [9]).

It is well known that $L^{p}(\mu, Y)$ is isometrically isomorphic to the norm completion $L^{p}(\mu) \widetilde{\otimes}_{\Delta_{p}} Y$ of $L^{p}(\mu) \otimes_{\Delta_{p}} Y$, where $\Delta_{p}$ denotes the induced Bochner norm (cf. [4, 5]). Chaney [1] characterized the Radon Nikodým property on $Y$ in terms of equality of $L^{p}(\mu) \widetilde{\otimes}_{\Delta_{p}} Y$ and the space of Dinculeanu's operators from $L^{q}(\mu)$ to $Y$ $\left(1<p<\infty\right.$ and $\left.\frac{1}{p}+\frac{1}{q}=1\right)$. These are the operators $T: L^{q}(\mu) \rightarrow Y$ for which $\infty>\|T\|_{q}:=\sup \left\{\sum_{i=1}^{n}\left\|\alpha_{i} T\left(\chi_{E_{i}}\right)\right\|_{Y}\right\}$, where the supremum is taken over all

Received by the editors August 1, 2007.

2000 Mathematics Subject Classification. Primary 46B28, 47B60, 60G48.

Key words and phrases. Bochner norm, Radon Nikodým property, convergent martingale, cone absolutely summing operator, 1-concave operator, Banach space, Banach lattice. 
simple functions $f=\sum_{i=1}^{n} \alpha_{i} \chi_{E_{i}}$ with $\chi_{E_{1}}, \ldots, \chi_{E_{n}} \in L^{q}(\mu), \alpha_{1}, \ldots, \alpha_{n} \in \mathbb{R}$ and $\|f\|_{q} \leq 1$ (cf. 6, 7, 8] ).

The aim of this paper is to extend Chaney's characterization by using Troitsky's [18] generalized notion of a martingale in a Banach lattice.

Chaney and Schaefer extended the Bochner norm to the tensor product of a Banach lattice $E$ and a Banach space $Y$; the norm $\|\cdot\|_{l}$, given by

$$
\|u\|_{l}=\inf \left\{\left\|\sum_{i=1}^{n}\right\| y_{i}\left\|\left|x_{i}\right|\right\|: u=\sum_{i=1}^{n} x_{i} \otimes y_{i}\right\}
$$

for all $u=\sum_{i=1}^{n} x_{i} \otimes y_{i} \in E \otimes Y$, coincides with the Bochner norm on $L^{p}(\mu) \otimes Y$ for all $\sigma$-finite measure spaces $(\Omega, \Sigma, \mu)$ and $1 \leq p<\infty$ (see [1], 17] and [1]).

Let $E$ be a Banach lattice and let $Y$ be a Banach space. Recall from [17, Chapter IV, §3] that a linear map $T: E \rightarrow Y$ is called cone absolutely summing if for every positive summable sequence $\left(x_{n}\right)$ in $E$, the sequence $\left(T x_{n}\right)$ is absolutely summable in $Y$. Such operators are known, in the terminology of Krivine, as 1concave operators (cf. [15, p. 45] and [13]). The space

$$
\mathcal{L}^{\text {cas }}(E, Y)=\{T: E \rightarrow Y: T \text { is cone absolutely summing }\}
$$

is a Banach space with respect to the norm defined by

$$
\|T\|_{\text {cas }}=\sup \left\{\sum_{i=1}^{n}\left\|T x_{i}\right\|: x_{1}, \ldots, x_{n} \in E_{+},\left\|\sum_{i=1}^{n} x_{i}\right\|=1, n \in \mathbb{N}\right\}
$$

for all $T \in \mathcal{L}^{\text {cas }}(E, Y)$. Cone absolutely summing operators generalize Dinculeanu's operators, mentioned above.

Cone absolutely summing operators extend the Chaney-Schaefer $l$-tensor product in the following sense: The canonical map $E^{*} \otimes_{l} Y \rightarrow \mathcal{L}^{\text {cas }}(E, Y)$ given by $\sum_{i=1}^{n} x_{i}^{*} \otimes y_{i}=: u \mapsto L_{u}$, where $L_{u} x=\sum_{i=1}^{n}\left\langle x, x_{i}^{*}\right\rangle y_{i}$ for all $x \in E$, is an isometry (cf. [17, Chapter IV, §7] and [1, 11, 14]). Here, $E^{*}$ denotes the continuous dual of $E$.

\section{Preliminaries}

We recall the abstract notions in [18] for a filtration and a martingale in a Banach space.

Definition 2.1. Let $E$ be a Banach lattice and $Y$ a Banach space.

(a) Let $\left(T_{i}\right)$ be a sequence of contractive projections on $Y$. If $T_{i \wedge j}=T_{i} T_{j}$ for each $i, j \in \mathbb{N}$, then $\left(T_{i}\right)$ is called a filtration on $Y$. In the case where $\left(T_{i}\right)$ is a filtration on a Banach lattice, we will always assume each $T_{i}$ to be positive.

(b) If $\left(T_{i}\right)$ is a filtration on $E$ with the property that each $\mathcal{R}\left(T_{i}\right)$ is a (closed) Riesz subspace of $E$, then $\left(T_{i}\right)$ will be called a $B L$-filtration on $E$ (this terminology is also used in [2]).

(c) If $\left(T_{i}\right)$ is a filtration on $E$ with each $T_{i}$ strictly positive (i.e., $T_{i}$ is positive and $\left.\left\{f \in E: T_{i}(|f|)=0\right\}=\{0\}\right)$, then $\left(T_{i}\right)$ is called a strictly positive filtration.

(d) If $\left(T_{i}\right)$ is a filtration on $Y$ and $\left(f_{i}\right) \subset Y$, then the pair $\left(f_{i}, T_{i}\right)$ is called a martingale in $Y$ if $T_{i} f_{j}=f_{i}$ for all $i \leq j$. 
(e) If $\left(f_{i}, T_{i}\right)$ is a martingale in $Y$, then $\left(f_{i}, T_{i}\right)$ is called fixed if there exists $f \in Y$ such that $f_{i}=T_{i} f$ for all $i \in \mathbb{N}$. In this case, $\left(f_{i}, T_{i}\right)$ is said to be fixed on $f$.

(f) If $\left(T_{i}\right)$ is a filtration on $E$, then $\left(f_{i}, T_{i}\right)$ is called a submartingale in $E$ if $T_{i} f_{j} \geq f_{i}$ for all $i \leq j$.

(g) Let $\left(T_{i}\right)$ be a filtration on $Y$. We say that $\left(T_{i}\right)$ is complemented in $Y$ if there exists a contractive projection $T_{\infty}: Y \rightarrow Y$ with $\mathcal{R}\left(T_{\infty}\right)=\overline{\bigcup_{i=1}^{\infty} \mathcal{R}\left(T_{i}\right)}$ and $T_{i} T_{\infty}=T_{\infty} T_{i}=T_{i}$ for all $i \in \mathbb{N}$.

If $(\Omega, \Sigma, \mu)$ is a finite measure space, $1 \leq p<\infty$ and $\left(\Sigma_{i}\right)$ an increasing sequence of $\sigma$-subalgebras of $\Sigma$, it follows that the sequence of conditional expectations $\left(\mathbb{E}\left(\cdot \mid \Sigma_{i}\right)\right)$ on $L^{p}(\mu)$ satisfies the above definition of a BL-filtration. Moreover, $\left(\mathbb{E}\left(\cdot \mid \Sigma_{i}\right)\right)$ is complemented by $\mathbb{E}\left(\cdot \mid \bigvee_{i=1}^{\infty} \Sigma_{i}\right): L^{p}(\mu) \rightarrow L^{p}(\mu)$, where $\bigvee_{i=1}^{\infty} \Sigma_{i}$ denotes the $\sigma$-algebra generated by $\bigcup_{i=1}^{\infty} \Sigma_{i}$.

In [17. Chapter III, $\S 11$, Proposition 11.5] it is shown that if $T: E \rightarrow E$ is a strictly positive projection on a Banach lattice $E$, then $\mathcal{R}(T)$ is a Banach sublattice of $E$. Consequently, every strictly positive filtration on $E$ is also a BL-filtration.

It is easily verified that if $\left(T_{i}\right)$ is a filtration on $E$, then the sequence of adjoint operators $\left(T_{i}^{*}\right)$ is a filtration on $E^{*}$. However, it is not clear that $\left(T_{i}^{*}\right)$ is a BLfiltration whenever $\left(T_{i}\right)$ is a BL-filtration. We need an additional definition.

Definition 2.2. Let $E$ be a Banach lattice with a non-empty quasi-interior $Q_{+}$. A filtration $\left(T_{i}\right)$ on $E$ is said to be quasi-interior preserving if $T_{i} Q_{+} \subset Q_{+}$for each $i \in \mathbb{N}$.

For background reading on quasi-interior points in Banach lattices, we refer the reader to [17, Chapter II, $\S 6]$.

Lemma 2.3. Let $E$ be a Banach lattice with a non-empty quasi-interior $Q_{+}$. If $T: E \rightarrow E$ is a positive projection, then $T Q_{+} \subset Q_{+}$if and only if there exists a quasi-interior point $0<e \in E_{+}$such that $T e=e$.

Proof. Note that $T \geq 0$ implies that $T$ is bounded. Suppose there exists $e \in Q_{+}$ such that $T e=e$ and let $q \in Q_{+}$. By [17, Chapter II, §6, Theorem 6.3], we have $\lim _{n \rightarrow \infty} T(n q \wedge e)=T(e)=e$. Also, $0 \leq T(n q \wedge e) \leq(n T q) \wedge e \leq e$ with $T(n q \wedge e) \uparrow$. Hence, $\lim _{n \rightarrow \infty}(n T q) \wedge e=e$. Now let $p \in E_{+}$. Using [17, Chapter II, $\S 6$, Theorem 6.3] again, we obtain

$$
p=\lim _{m \rightarrow \infty}(m e) \wedge p=\lim _{m \rightarrow \infty}\left(m \lim _{n \rightarrow \infty}(n T q) \wedge e\right) \wedge p=\lim _{m \rightarrow \infty} \lim _{n \rightarrow \infty}(m(n T q) \wedge e) \wedge p .
$$

As $(m(n T q) \wedge e) \wedge p \leq(m n T q) \wedge p \leq p$ for each $n, m \in \mathbb{N}$, it follows that

$$
\lim _{m \rightarrow \infty} \lim _{n \rightarrow \infty}(m n T q) \wedge p=p
$$

Thus, $T q \in Q_{+}$by [17, Chapter II, $\S 6$, Theorem 6.3], i.e. $T Q_{+} \subset Q_{+}$. The converse is trivial.

The spaces $L^{p}(\mu), 1 \leq p<\infty$, have non-empty quasi-interior (e.g., 1 is a quasiinterior point of $\left.L^{p}(\mu)\right)$ and if $\left(\Sigma_{i}\right)$ is an increasing sequence of $\sigma$-subalgebras of $\Sigma$, then the sequence of conditional expectations $\left(\mathbb{E}\left(\cdot \mid \Sigma_{i}\right)\right)$ is a filtration with $\mathbb{E}\left(\mathbf{1} \mid \Sigma_{i}\right)=\mathbf{1}$ for each $i \in \mathbb{N}$. By the above lemma, $\left(\mathbb{E}\left(\cdot \mid \Sigma_{i}\right)\right)$ is a filtration that is quasi-interior preserving. 
Proposition 2.4. Suppose that $E$ is a Banach lattice possessing a non-empty quasiinterior $Q_{+}$and $T: E \rightarrow E$ is a bounded linear operator. Then $T Q_{+} \subset Q_{+}$if and only if $T^{*}: E^{*} \rightarrow E^{*}$ is strictly positive.

Proof. By [17, Chapter II, $\S 6$, Theorem 6.3], $q \in Q_{+}$if and only if $\left\langle q, f^{*}\right\rangle>0$ for all $0<f^{*} \in E^{*}$. With this in mind, assume that $T Q_{+} \subset Q_{+}$. Since $Q_{+}$is dense in $E_{+}$, it follows that $T \geq 0$, which implies $T^{*} \geq 0$. To show strict positivity, suppose that $T^{*} f^{*}=0$ for some $f^{*} \in E_{+}^{*}$. Then $0=\left\langle q, T^{*} f^{*}\right\rangle=\left\langle T q, f^{*}\right\rangle$ for $q \in Q_{+}$. Since $T q \in Q_{+}$, it follows that $f^{*}=0$. Conversely, if $T^{*}$ is strictly positive, then for all $0<f^{*} \in E^{*}$ we have $T^{*} f^{*}>0$. Thus, for $q \in Q_{+}$, it follows that $\left\langle T q, f^{*}\right\rangle=\left\langle q, T^{*} f^{*}\right\rangle>0$ for all $f^{*} \in E^{*}$. Consequently, $T q \in Q_{+}$.

Corollary 2.5. Suppose that $E$ is a Banach lattice possessing non-empty quasiinterior. Then for any quasi-interior preserving filtration $\left(T_{i}\right)$ on $E$, we have that $\left(T_{i}^{*}\right)$ is a $B L$-filtration on $E^{*}$.

The following convergence result was shown in [2].

Proposition 2.6. Let $Y$ be a Banach space and $\left(T_{i}\right)$ a filtration on $Y$. Then the following statements hold:

(a) $f \in \overline{\bigcup_{i=1}^{\infty} \mathcal{R}\left(T_{i}\right)}$ if and only if $\left\|T_{i} f-f\right\| \rightarrow 0$ as $i \rightarrow \infty$.

(b) If $\left(f_{i}\right)$ is a martingale relative to $\left(T_{i}\right)$, then $\left(f_{i}\right)$ converges to $f$ if and only if $f \in \overline{\bigcup_{i=1}^{\infty} \mathcal{R}\left(T_{i}\right)}$ and $f_{i}=T_{i} f$ for all $i \in \mathbb{N}$.

Consider the case where a filtration $\left(T_{i}\right)$ on a Banach lattice $E$ is complemented in $E$ by a contractive projection $T_{\infty}: E \rightarrow E$. Since each $T_{i}$ is assumed to be positive, it follows that $T_{\infty}$ is also positive. Indeed, if $f \in E_{+}$, then $T_{i} f \in E_{+}$for each $i \in \mathbb{N}$. Thus, Proposition 2.6 implies $\lim _{i \rightarrow \infty} T_{i} f=\lim _{i \rightarrow \infty} T_{i} T_{\infty} f=T_{\infty} f \geq 0$.

Definition 2.7. Let $Y$ be a Banach space and $\left(T_{i}\right)$ a filtration on $Y$.

(a) Define the space of norm bounded martingales as

$$
\mathcal{M}\left(Y, T_{i}\right)=\left\{\left(f_{i}, T_{i}\right) \text { a martingale in } Y: \sup _{i}\left\|f_{i}\right\|<\infty\right\},
$$

together with the norm defined by $\left\|\left(f_{i}, T_{i}\right)\right\|=\sup _{i}\left\|f_{i}\right\|$ for all $\left(f_{i}, T_{i}\right) \in$ $\mathcal{M}\left(Y, T_{i}\right)$.

(b) Define the space of norm convergent martingales by

$$
\mathcal{M}_{\mathrm{nc}}\left(Y, T_{i}\right)=\left\{\left(f_{i}, T_{i}\right) \in \mathcal{M}\left(Y, T_{i}\right):\left(f_{i}\right) \text { is norm convergent in } Y\right\} .
$$

(c) Define the space of fixed martingales by

$$
\mathcal{M}_{\mathrm{f}}\left(Y, T_{i}\right)=\left\{\left(f_{i}, T_{i}\right) \in \mathcal{M}\left(Y, T_{i}\right): \exists f \in Y \text { so that } T_{i} f=f_{i} \forall i \in \mathbb{N}\right\} .
$$

It is easily shown that $\mathcal{M}\left(Y, T_{i}\right)$ and $\mathcal{M}_{\mathrm{nc}}\left(Y, T_{i}\right)$ are Banach spaces. By Proposition 2.6 (b), we have the inclusions $\mathcal{M}_{\mathrm{nc}}\left(Y, T_{i}\right) \subset \mathcal{M}_{\mathrm{f}}\left(Y, T_{i}\right) \subset \mathcal{M}\left(Y, T_{i}\right)$. Note that if $\left(T_{i}\right)$ is a complemented filtration on $Y$, then $\mathcal{M}_{\mathrm{nc}}\left(Y, T_{i}\right)=\mathcal{M}_{\mathrm{f}}\left(Y, T_{i}\right)$. If $\left(f_{i}, T_{i}\right)$ is fixed, then it is also fixed on an element in $\overline{\bigcup_{i=1}^{\infty} \mathcal{R}\left(T_{i}\right)}$ and thus convergent by Proposition 2.6.

In the case where $Y$ is reflexive, Troitsky showed in [18, Corollary 18] that $\mathcal{M}_{\mathrm{f}}\left(Y, T_{i}\right)=\mathcal{M}\left(Y, T_{i}\right)$. If $\left(T_{i}\right)$ is also complemented in $Y$, then $\mathcal{M}_{\mathrm{nc}}\left(Y, T_{i}\right)=$ $\mathcal{M}_{\mathrm{f}}\left(Y, T_{i}\right)=\mathcal{M}\left(Y, T_{i}\right)$.

For a BL-filtration $\left(T_{i}\right)$ on a Banach lattice $E$, [2, Proposition 3.7] implies that $\mathcal{M}_{\mathrm{nc}}\left(E, T_{i}\right)$ is a Banach lattice with respect to the ordering defined by $\left(f_{i}, T_{i}\right) \geq$ 
$0 \Leftrightarrow f_{i} \geq 0$ for all $i \in \mathbb{N}$. Moreover, it is shown that $\mathcal{M}_{\mathrm{nc}}\left(E, T_{i}\right)$ is Riesz isometric to $\overline{\bigcup_{i=1}^{\infty} \mathcal{R}\left(T_{i}\right)}$.

In the case where $E$ is a KB-space and $\left(T_{i}\right)$ is a filtration on $E$, Troitsky showed in [18, Theorem 7] that $\mathcal{M}\left(E, T_{i}\right)$ is also a Banach lattice under this ordering. Moreover, Troitsky showed the following, which is key to our main result (cf. 18, p. 446]).

Proposition 2.8. Let $E$ be a KB-space and $\left(T_{i}\right)$ a filtration on $E$. If $\left(s_{i}, T_{i}\right)$ is a norm bounded submartingale in $E$, then there exists a unique least martingale $\left(f_{i}, T_{i}\right) \in \mathcal{M}\left(E, T_{i}\right)$ such that $s_{i} \leq f_{i}$ for each $i \in \mathbb{N}$. Moreover, $\left\|\left(f_{i}, T_{i}\right)\right\| \leq$ $\left\|\left(s_{i}, T_{i}\right)\right\|$.

The notion of a filtration $\left(T_{i}\right)$ on a Banach lattice $E$ can be extended to the $l$ tensor product $E \widetilde{\otimes}_{l} Y$ for any Banach space $Y$. Indeed, it follows from the proof of [2. Lemma 4.2] that $\left(T_{i} \otimes_{l} \mathrm{id}_{\mathrm{Y}}\right)$ is a sequence of commuting contractive projections on $E \widetilde{\otimes}_{l} Y$ with increasing range. This extension is consistent with a classical filtration on the Lebesgue-Bochner spaces.

\section{Cone absolutely summing martingales}

To characterize the Radon Nikodým property, we require a fair amount of preparation. We first consider a canonical filtration on the space of cone absolutely summing operators from a Banach lattice $E$ to a Banach space $Y$.

Proposition 3.1. Let $E$ be a Banach lattice and $Y$ a Banach space. Suppose that $\left(T_{i}\right)$ is a BL-filtration on $E$. Then the sequence $\left(\widehat{T}_{i}\right)$ of maps $\widehat{T}_{i}: \mathcal{L}^{\text {cas }}(E, Y) \rightarrow$ $\mathcal{L}^{\text {cas }}(E, Y)$, defined by $\widehat{T}_{i} F=F \circ T_{i}$ for each $F \in \mathcal{L}^{\operatorname{cas}}(E, Y)$ and $i \in \mathbb{N}$, is a sequence of contractive projections on $\mathcal{L}^{\text {cas }}(E, Y)$ with $\widehat{T}_{i \wedge j}=\widehat{T}_{i} \widehat{T}_{j}$.

Proof. Since $\left(T_{i}\right)$ is a filtration, $F \circ T_{i} \in \mathcal{L}^{\text {cas }}(E, Y)$ and $\widehat{T}_{i}$ is a well-defined, linear projection for each $i \in \mathbb{N}$. It also follows from

$$
\begin{aligned}
\left\|\widehat{T}_{i} F\right\|_{\text {cas }} & =\sup \left\{\sum_{j=1}^{n}\left\|F T_{i} x_{j}\right\|:\left(x_{j}\right)_{i=1}^{n} \subset E_{+},\left\|\sum_{j=1}^{n} x_{j}\right\| \leq 1\right\} \\
& =\sup \left\{\sum_{j=1}^{n}\left\|F x_{j}\right\|:\left(x_{j}\right)_{i=1}^{n} \subset \mathcal{R}\left(T_{i}\right)_{+},\left\|\sum_{j=1}^{n} x_{j}\right\| \leq 1\right\} \\
& \leq \sup \left\{\sum_{j=1}^{n}\left\|F x_{j}\right\|:\left(x_{j}\right)_{i=1}^{n} \subset E_{+},\left\|\sum_{j=1}^{n} x_{j}\right\| \leq 1\right\} \\
& =\|F\|_{\text {cas }}
\end{aligned}
$$

that each $\widehat{T}_{i}$ is bounded and $\sup _{i \in \mathbb{N}}\left\|\widehat{T}_{i}\right\|=1$. Moreover,

$$
\widehat{T}_{i} \widehat{T}_{j} F=F \circ T_{j} \circ T_{i}=F \circ T_{i \wedge j}=\widehat{T}_{i \wedge j} F
$$

for each $F \in \mathcal{L}^{\operatorname{cas}}(E, Y)$ and $i, j \in \mathbb{N}$. This completes the proof.

In view of the above proposition, we are justified in making the following definition. 
Definition 3.2. Let $E$ be a Banach lattice and $Y$ a Banach space. Suppose that $\left(T_{i}\right)$ is a BL-filtration on $E$. Then $\left(\widehat{T}_{i}\right)$, as defined in Proposition 3.1, is called the filtration on $\mathcal{L}^{\text {cas }}(E, Y)$ induced by $\left(T_{i}\right)$.

We exhibit a known characterization of cone absolutely summing operators.

Lemma 3.3. Let $E$ be a Banach lattice, $Y$ a Banach space and $l>0$. For any bounded operator $T: E \rightarrow Y$ the following statements are equivalent:

(a) $T$ is cone absolutely summing with $\|T\|_{\text {cas }} \leq l$.

(b) There exists $x_{T}^{*} \in E_{+}^{*}$ so that $\left\|x_{T}^{*}\right\| \leq l$ and $\|T x\| \leq\left\langle|x|, x_{T}^{*}\right\rangle$ for all $x \in E$.

(c) There exist an $A L$-space $L, 0 \leq T_{1} \in \mathcal{L}(E, L)$ and $T_{2} \in \mathcal{L}(L, Y)$ such that $T=T_{2} \circ T_{1}$, where $\left\|T_{1}\right\| \leq l$ and $\left\|T_{2}\right\| \leq 1$.

In the case where $E$ is separable, we may take $L=L^{1}(\mu)$ in $(c)$, where $(\Omega, \Sigma, \mu)$ is a finite measure space.

The proof of the equivalence of (a), (b) and (c) in the above lemma may be found in [17, Chapter IV, $\S 3$, Proposition 3.3]. However, the last part of the lemma requires a proof: Assume $E$ is separable. Then, by [17, Chapter II, $\S 6$, Proposition 6.2], there exists a quasi-interior point $0 \leq e \in E$. By construction, the map $T_{1}: E \rightarrow L$ is a Riesz homomorphism with dense range (see [17, p. 243, §3]). Thus, it follows by [17, Chapter II, $\S 6$, Proposition 6.4] that $T_{1} e$ is a quasi-interior point of $L$. Hence, $T_{1} e$ is also a weak order unit of $L$. It follows by Kakutani's representation theorem for $A L$-spaces (cf. [12 or [15, Theorem 1.b.2]) that $L$ is Riesz and isometrically isomorphic to $L^{1}(\mu)$, where $(\Omega, \Sigma, \mu)$ may be chosen to be finite.

For our next result, we recall that the functional $x_{T}^{*} \in E_{+}^{*}$ in Lemma 3.3(b) is the extension of the additive map $\rho_{T}: E_{+} \rightarrow E_{+}$, defined by

$$
\rho_{T}(x)=\sup \left\{\sum_{i=1}^{\infty}\left\|T x_{i}\right\|:\left(x_{i}\right) \in\left(\ell^{1} \widetilde{\otimes}_{\varepsilon} E\right)_{+}, \sum_{i=1}^{\infty} x_{i}=x\right\}
$$

for each $x \in E_{+}$(cf. [17, Chapter IV, $\S 2$, Theorem 2.7]). Here, $\ell^{1} \widetilde{\otimes}_{\varepsilon} E$ denotes the space of unconditionally summable sequences in $E$.

Proposition 3.4. Let $E$ be a Banach lattice with order continuous dual and $Y$ a Banach space. Suppose that $\left(T_{i}\right)$ is a BL-filtration on $E$ and $\left(\widehat{T}_{i}\right)$ is the filtration on $\mathcal{L}^{\text {cas }}(E, Y)$ induced by $\left(T_{i}\right)$. If $\left(F_{i}, \widehat{T}_{i}\right) \in \mathcal{M}\left(\mathcal{L}^{\text {cas }}(E, Y), \widehat{T}_{i}\right)$, then there exists $0 \leq\left(f_{i}^{*}, T_{i}^{*}\right) \in \mathcal{M}\left(E^{*}, T_{i}^{*}\right)$ such that $\left\|F_{i} x\right\| \leq\left\langle|x|, f_{i}^{*}\right\rangle$ for each $x \in E$ and $i \in \mathbb{N}$. Moreover, $\sup _{i \in \mathbb{N}}\left\|f_{i}^{*}\right\| \leq \sup _{i \in \mathbb{N}}\left\|F_{i}\right\|_{\text {cas }}$.

Proof. By Lemma 3.3 there exists, for each $F_{i}$, a positive functional $x_{F_{i}}^{*} \in E^{*}$ with $\left\|x_{F_{i}}^{*}\right\| \leq \sup _{i \in \mathbb{N}}\left\|F_{i}\right\|_{\text {cas }}:=l$ and $\left\|F_{i} x\right\| \leq\left\langle|x|, x_{F_{i}}^{*}\right\rangle$ for each $x \in E$ and $i \in \mathbb{N}$. Define $s_{i}^{*} \in E_{+}^{*}$ by $\left\langle x, s_{i}^{*}\right\rangle=\left\langle T_{i} x, x_{F_{i}}^{*}\right\rangle$ for each $x \in E$ and $i \in \mathbb{N}$. Then, $\sup _{i \in \mathbb{N}}\left\|s_{i}^{*}\right\| \leq l$ and, since $x_{F_{i}}^{*} \geq 0$, we get

$$
\left\|F_{i} x\right\|=\left\|\widehat{T}_{i} F_{i} x\right\|=\left\|F_{i} T_{i} x\right\| \leq\left\langle\left|T_{i} x\right|, x_{F_{i}}^{*}\right\rangle \leq\left\langle T_{i}|x|, x_{F_{i}}^{*}\right\rangle=\left\langle|x|, s_{i}^{*}\right\rangle
$$


for all $x \in E$ and $i \in \mathbb{N}$. We now show that $\left(s_{i}^{*}, T_{i}^{*}\right)$ is a submartingale. Let $i \leq j$ and $x \in E_{+}$. Then,

$$
\begin{aligned}
\left\langle x, T_{i}^{*} s_{j}^{*}\right\rangle & =\left\langle T_{i} x, s_{j}^{*}\right\rangle=\left\langle T_{j} T_{i} x, x_{F_{j}}^{*}\right\rangle=\left\langle T_{i} x, x_{F_{j}}^{*}\right\rangle \\
& =\sup \left\{\sum_{n=1}^{\infty}\left\|F_{j} x_{n}\right\|:\left(x_{n}\right) \in\left(\ell^{1} \widetilde{\otimes}_{\varepsilon} E\right)_{+}, \sum_{n=1}^{\infty} x_{n}=T_{i} x\right\} \\
& \geq \sup \left\{\sum_{n=1}^{\infty}\left\|F_{j} x_{n}\right\|:\left(x_{n}\right) \in\left(\ell^{1} \widetilde{\otimes}_{\varepsilon} \mathcal{R}\left(T_{i}\right)\right)_{+}, \sum_{n=1}^{\infty} x_{n}=T_{i} x\right\}
\end{aligned}
$$

as $\left(\ell^{1} \widetilde{\otimes}_{\varepsilon} \mathcal{R}\left(T_{i}\right)\right)_{+} \subset\left(\ell^{1} \widetilde{\otimes}_{\varepsilon} E\right)_{+}$. Since $T_{i}$ is a projection, it follows for $x \in E$ that $x \in \mathcal{R}\left(T_{i}\right)$ if and only if $x=T_{i} x$. In addition, the positivity of $T_{i}$ implies $\left(T_{i} x_{n}\right) \in$ $\left(\ell^{1} \widetilde{\otimes}_{\varepsilon} \mathcal{R}\left(T_{i}\right)\right)_{+}$for all $\left(x_{i}\right) \in\left(\ell^{1} \widetilde{\otimes}_{\varepsilon} E\right)_{+}$. Consequently, for $\left(x_{n}\right) \in\left(\ell^{1} \widetilde{\otimes}_{\varepsilon} E\right)_{+}$, we have $\left(x_{n}\right) \in\left(\ell^{1} \widetilde{\otimes}_{\mathcal{E}} \mathcal{R}\left(T_{i}\right)\right)_{+}$if and only if $\left(x_{n}\right)=\left(T_{i} x_{n}\right)$. Thus,

$$
\begin{aligned}
& \sup \left\{\sum_{n=1}^{\infty}\left\|F_{j} x_{n}\right\|:\left(x_{n}\right) \in\left(\ell^{1} \widetilde{\otimes}_{\varepsilon} \mathcal{R}\left(T_{i}\right)\right)_{+}, \sum_{n=1}^{\infty} x_{n}=T_{i} x\right\} \\
& =\sup \left\{\sum_{n=1}^{\infty}\left\|F_{j} T_{i} x_{n}\right\|:\left(x_{n}\right) \in\left(\ell^{1} \widetilde{\otimes}_{\varepsilon} E\right)_{+}, \sum_{n=1}^{\infty} x_{n}=T_{i} x\right\} \\
& =\sup \left\{\sum_{n=1}^{\infty}\left\|\widehat{T}_{i} F_{j} x_{n}\right\|:\left(x_{n}\right) \in\left(\ell^{1} \widetilde{\otimes}_{\varepsilon} E\right)_{+}, \sum_{n=1}^{\infty} x_{n}=T_{i} x\right\} \\
& =\sup \left\{\sum_{n=1}^{\infty}\left\|F_{i} x_{n}\right\|:\left(x_{n}\right) \in\left(\ell^{1} \widetilde{\otimes}_{\varepsilon} E\right)_{+}, \sum_{n=1}^{\infty} x_{n}=T_{i} x\right\} \\
& =\left\langle T_{i} x, x_{F_{i}}^{*}\right\rangle=\left\langle x, s_{i}^{*}\right\rangle .
\end{aligned}
$$

Since $s_{i}^{*}(x) \leq T_{i}^{*} s_{j}^{*}(x)$ for all $x \in E_{+}$, it follows that $s_{i}^{*} \leq T_{i}^{*} s_{j}^{*}$. Consequently, $\left(s_{i}^{*}, T_{i}^{*}\right)$ is a submartingale. Since $E^{*}$ is order continuous, it follows that $E^{*}$ is a KBspace (cf. [16, Theorem 2.4.14]). Thus, by Proposition 2.8, there exists a unique least martingale $0 \leq\left(f_{i}^{*}, T_{i}^{*}\right) \in \mathcal{M}\left(E^{*}, T_{i}^{*}\right)$ that dominates the submartingale $\left(s_{i}^{*}, T_{i}^{*}\right)$, with $\sup _{i \in \mathbb{N}}\left\|f_{i}^{*}\right\| \leq \sup _{i \in \mathbb{N}}\left\|s_{i}^{*}\right\| \leq l=\sup _{i \in \mathbb{N}}\left\|F_{i}\right\|_{\text {cas. }}$. Hence,

$$
\left\|F_{i} x\right\| \leq\left\langle|x|, s_{i}^{*}\right\rangle \leq\left\langle|x|, f_{i}^{*}\right\rangle
$$

for all $x \in E$, and the proof is complete.

Theorem 3.5. Let $E$ be a Banach lattice with order continuous dual and $Y$ a Banach space. Suppose that $\left(T_{i}\right)$ is a BL-filtration on $E$ and $\left(\widehat{T}_{i}\right)$ is the filtration on $\mathcal{L}^{\text {cas }}(E, Y)$ induced by $\left(T_{i}\right)$. Then $\mathcal{M}_{\mathrm{f}}\left(\mathcal{L}^{\text {cas }}(E, Y), \widehat{T}_{i}\right)=\mathcal{M}\left(\mathcal{L}^{\text {cas }}(E, Y), \widehat{T}_{i}\right)$.

Proof. The inclusion $\mathcal{M}_{\mathrm{f}}\left(\mathcal{L}^{\text {cas }}(E, Y), \widehat{T}_{i}\right) \subset \mathcal{M}\left(\mathcal{L}^{\text {cas }}(E, Y), \widehat{T}_{i}\right)$ is obvious. For the reverse inclusion, let $\left(F_{i}, \widehat{T}_{i}\right) \in \mathcal{M}\left(\mathcal{L}^{\text {cas }}(E, Y), \widehat{T}_{i}\right)$. By Proposition 3.4, there exists $0 \leq\left(f_{i}^{*}, T_{i}^{*}\right) \in \mathcal{M}\left(E^{*}, T_{i}^{*}\right)$ such that $\sup _{i \in \mathbb{N}}\left\|f_{i}^{*}\right\| \leq \sup _{i \in \mathbb{N}}\left\|F_{i}\right\|_{\text {cas }}$ and $\left\|F_{i} x\right\| \leq\left\langle|x|, f_{i}^{*}\right\rangle$ for each $x \in E$ and $i \in \mathbb{N}$. Let $\sup _{i \in \mathbb{N}}\left\|f_{i}^{*}\right\|:=K$ and define $f^{*}: \bigcup_{i=1}^{\infty} \mathcal{R}\left(T_{i}\right) \rightarrow \mathbb{R}$ by $\left\langle x, f^{*}\right\rangle=\lim _{i \rightarrow \infty}\left\langle x, f_{i}^{*}\right\rangle$ for each $x \in \bigcup_{i=1}^{\infty} \mathcal{R}\left(T_{i}\right)$. Observe that $f^{*}$ is well defined, as for $x \in \bigcup_{i=1}^{\infty} \mathcal{R}\left(T_{i}\right)$ there exists $i \in \mathbb{N}$ such that $x \in \mathcal{R}\left(T_{i}\right)$. Consequently, $i \leq j$ implies $\left\langle x, f_{i}^{*}\right\rangle=\left\langle x, T_{i}^{*} f_{j}^{*}\right\rangle=\left\langle T_{i} x, f_{j}^{*}\right\rangle=\left\langle x, f_{j}^{*}\right\rangle$. Thus, $\left\langle x, f^{*}\right\rangle=\lim _{i \rightarrow \infty}\left\langle x, f_{i}^{*}\right\rangle$ exists for each $x \in \bigcup_{i=1}^{\infty} \mathcal{R}\left(T_{i}\right)$. Evidently, $f^{*}$ is positive, linear and the inequality $\left|\left\langle x, f^{*}\right\rangle\right|=\lim _{i \rightarrow \infty}\left|\left\langle x, f_{i}^{*}\right\rangle\right| \leq \lim _{i \rightarrow \infty}\left\|f_{i}^{*}\right\|\|x\|=K\|x\|$ shows that $f^{*}$ is also bounded with norm $\left\|f^{*}\right\| \leq K$. 
Now define a map $F: \bigcup_{i=1}^{\infty} \mathcal{R}\left(T_{i}\right) \rightarrow Y$ by $F x=\lim _{i \rightarrow \infty} F_{i} x$ for each $x \in$ $\bigcup_{i=1}^{\infty} \mathcal{R}\left(T_{i}\right)$. The map $F$ is well defined because, for each $x \in \bigcup_{i=1}^{\infty} \mathcal{R}\left(T_{i}\right)$, there is some $i \in \mathbb{N}$ for which $x \in \mathcal{R}\left(T_{i}\right)$. Thus, $i \leq j$ implies $F_{i} x=\widehat{T}_{i} F_{j} x=F_{j} T_{i} x=F_{j} x$ so that $F x=\lim _{i \rightarrow \infty} F_{i} x$ exists for each $x \in \bigcup_{i=1}^{\infty} \mathcal{R}\left(T_{i}\right)$. It is now evident that $F$ is linear. Moreover, since $\bigcup_{i=1}^{\infty} \mathcal{R}\left(T_{i}\right)$ is a Riesz subspace of $E$, we have

$$
\|F x\|=\lim _{i \rightarrow \infty}\left\|F_{i} x\right\| \leq \lim _{i \rightarrow \infty}\left\langle|x|, f_{i}^{*}\right\rangle=\left\langle|x|, f^{*}\right\rangle \leq K\|x\|
$$

for all $x \in \bigcup_{i=1}^{\infty} \mathcal{R}\left(T_{i}\right)$. Thus, $F$ is bounded. Let $\bar{f}^{*}$ and $\bar{F}$ denote the unique continuous extensions of $f^{*}$ and $F$ respectively to the Banach sublattice $\overline{\bigcup_{i=1}^{\infty} \mathcal{R}\left(T_{i}\right)}$ of $E$. Then we have $\|\bar{F} x\| \leq\left\langle|x|, \bar{f}^{*}\right\rangle$ for all $x \in \overline{\bigcup_{i=1}^{\infty} \mathcal{R}\left(T_{i}\right)}$. Consequently, Lemma 3.3 implies $\bar{F} \in \mathcal{L}^{\text {cas }}\left(\overline{\bigcup_{i=1}^{\infty} \mathcal{R}\left(T_{i}\right)}, Y\right)$. By [17, Chapter IV, §3, Proposition 3.9], $\bar{F}$ possesses an extension $\bar{F}_{\infty} \in \mathcal{L}^{\text {cas }}(E, Y)$ with $\|\bar{F}\|_{\text {cas }}=\left\|\bar{F}_{\infty}\right\|_{\text {cas }}$. Finally, $\widehat{T}_{i} \bar{F}_{\infty} x=F T_{i} x=\lim _{j \rightarrow \infty} F_{j} T_{i} x=\lim _{j \rightarrow \infty} \widehat{T}_{i} F_{j} x=F_{i} x$ for all $x \in E$ and $i \in \mathbb{N}$. Thus, $\left(F_{i}, \widehat{T}_{i}\right) \in \mathcal{M}_{\mathrm{f}}\left(\mathcal{L}^{\operatorname{cas}}(E, Y), \widehat{T}_{i}\right)$.

We continue our preparations with the next lemma, which is a simple restatement of well-known facts about order continuity of the norm in dual Banach lattices.

Lemma 3.6. Let $E$ be a Banach lattice such that $E^{*}$ has order continuous norm. If $T: E \rightarrow \ell^{1}$ is a positive linear operator, then $T$ is compact.

Proof. Let $T: E \rightarrow \ell^{1}$ be a positive operator. Denote the restriction of $T^{*}$ to $c_{0}$ by $\left.T^{*}\right|_{c_{0}}$. Then $\left.T^{*}\right|_{c_{0}}: c_{0} \rightarrow E^{*}$ is positive. But $E^{*}$ is a KB-space by 16 , Theorem 2.4.14]; thus, $\left.T^{*}\right|_{c_{0}}$ is weakly compact (cf. [17, Chapter II, $\S 5$, Proposition 5.15]). Consequently, $\left(\left.T^{*}\right|_{c_{0}}\right)^{*}: E^{* *} \rightarrow \ell^{1}$ is compact because $\ell^{1}$ has the Schur property. Hence, $T=\left.\left(T^{*} \mid c_{c_{0}}\right)^{*}\right|_{E}$ is compact.

Lastly, we need the following characterization of the $l$-tensor product, which is shown in [14, Theorem 5.2].

Theorem 3.7. Let $E$ be a Banach lattice, $Y$ a Banach space and $T \in \mathcal{L}(E, Y)$. Then $T \in E^{*} \widetilde{\otimes}_{l} Y$ if and only if there exist $0 \leq S \in \mathcal{L}\left(E, \ell^{1}\right)$ and $R \in \mathcal{L}\left(\ell^{1}, Y\right)$ such that $S$ is compact and $T=R \circ S$. Further, $\|T\|_{\text {cas }}=\inf \|R\|\|S\|_{r}$, where the infimum is taken over all such factorizations of $T$.

We are now prepared to characterize the Radon Nikodým property:

Theorem 3.8. Let $Y$ be a Banach space. Then the following statements are equivalent:

(a) $Y$ has the Radon Nikodym property.

(b) $E^{*} \widetilde{\otimes}_{l} Y=\mathcal{L}^{\text {cas }}(E, Y)$ for all separable Banach lattices $E$ with order continuous dual.

(c) $\mathcal{M}\left(E^{*} \widetilde{\otimes}_{l} Y, T_{i}^{*} \otimes_{l} \operatorname{id}_{Y}\right)=\mathcal{M}_{\mathrm{f}}\left(E^{*} \widetilde{\otimes}_{l} Y, T_{i}^{*} \otimes_{l} \operatorname{id}_{Y}\right)$ for all separable Banach lattices $E$ with order continuous dual and all BL-filtrations $\left(T_{i}\right)$ on $E$.

(d) $\mathcal{M}\left(E \widetilde{\otimes}_{l} Y, T_{i} \otimes_{l} \mathrm{id}_{Y}\right)=\mathcal{M}_{\mathrm{nc}}\left(E \widetilde{\otimes}_{l} Y, T_{i} \otimes_{l} \mathrm{id}_{Y}\right)$ for all separable reflexive Banach lattices $E$ and all complemented, quasi-interior preserving $B L$ filtrations $\left(T_{i}\right)$ on $E$.

(e) $\mathcal{M}\left(E \widetilde{\otimes}_{l} Y, T_{i} \otimes_{l} \operatorname{id}_{Y}\right)=\mathcal{M}\left(E, T_{i}\right) \widetilde{\otimes}_{l} Y$ for all separable reflexive Banach lattices $E$ and all complemented, quasi-interior preserving BL-filtrations $\left(T_{i}\right)$ on $E$. 
Proof. (a) $\Rightarrow$ (b) Let $E$ be a separable Banach lattice with order continuous dual. Let $T \in \mathcal{L}^{\operatorname{cas}}(E, Y)$. By Lemma 3.3 , there exist a finite measure space $(\Omega, \Sigma, \mu)$ and operators $0 \leq T_{1} \in \mathcal{L}\left(E, L^{1}(\mu)\right)$ and $T_{2} \in \mathcal{L}\left(L^{1}(\mu), Y\right)$ such that $T=T_{2} \circ T_{1}$ where $\left\|T_{1}\right\| \leq\|T\|_{\text {cas }}$ and $\left\|T_{2}\right\| \leq 1$. Since $Y$ has the Radon Nikodým property, the LewisStegall Theorem (cf. [5, Chapter III, $\S 1$, Theorem 8]) guarantees the existence of operators $0 \leq S_{1} \in \mathcal{L}\left(L^{1}(\mu), \ell^{1}\right)$ and $S_{2} \in \mathcal{L}\left(\ell^{1}, Y\right)$ such that $T_{2}=S_{2} \circ S_{1}$. Since $E^{*}$ is order continuous, the positive operator $S_{1} \circ T_{1}: E \rightarrow \ell^{1}$ is compact by Lemma 3.6. Hence, $T \in E^{*} \widetilde{\otimes}_{l} Y$ by Theorem 3.7 .

(b) $\Rightarrow$ (c) Suppose $E$ is a separable Banach lattice with order continuous dual and $\left(T_{i}\right)$ is a filtration on $E$. Let $\left(f_{i}, T_{i}^{*} \otimes_{l} \operatorname{id}_{Y}\right) \in \mathcal{M}\left(E^{*} \widetilde{\otimes}_{l} Y, T_{i}^{*} \otimes \operatorname{id}_{Y}\right)$. By (b), $E^{*} \widetilde{\otimes}_{l} Y$ is isometric to $\mathcal{L}^{\text {cas }}(E, Y)$ under the continuous extension of the canonical isometry $E^{*} \otimes_{l} Y \rightarrow \mathcal{L}^{\text {cas }}(E, Y)$, given by $u \mapsto L_{u}$, where $L_{u}=\sum_{i=1}^{n}\left\langle\cdot, x_{i}^{*}\right\rangle y_{i}$ for $u=\sum_{i=1}^{n} x_{i}^{*} \otimes y_{i}$. Let $\left(F_{i}\right) \subset \mathcal{L}^{\text {cas }}(E, Y)$ be the sequence corresponding to the martingale $\left(f_{i}, T_{i}^{*} \otimes_{l} \operatorname{id}_{\mathrm{Y}}\right)$. Observe that, for $u=\sum_{k=1}^{n} x_{k}^{*} \otimes y_{k} \in E^{*} \otimes Y$, we have

$$
\left(T_{i}^{*} \otimes \operatorname{id}_{Y}\right) u \mapsto \sum_{i=1}^{k}\left\langle\cdot, T_{i}^{*} x_{k}^{*}\right\rangle y_{k}=L_{u} \circ T_{i}=\widehat{T}_{i} L_{u}
$$

for all $i \in \mathbb{N}$. It follows that $\left(F_{i}, \widehat{T}_{i}\right) \in \mathcal{M}\left(\mathcal{L}^{\text {cas }}(E, Y), \widehat{T}_{i}\right)$. By Theorem 3.5. there exists $F_{\infty} \in \mathcal{L}^{\text {cas }}(E, Y)$ such that $\widehat{T}_{i} F_{\infty}=F_{i}$ for each $i \in \mathbb{N}$. Consequently, $\left(f_{i}, T_{i}^{*} \otimes_{l} \operatorname{id}_{Y}\right) \in \mathcal{M}_{\mathrm{f}}\left(E^{*} \widetilde{\otimes}_{l} Y, T_{i}^{*} \otimes_{l} \operatorname{id}_{Y}\right)$.

$(\mathrm{c}) \Rightarrow(\mathrm{d})$ Since $E$ is a separable reflexive Banach lattice, $E$ has non-empty quasiinterior, $E^{* *}$ is order continuous and $E^{*}$ is separable (cf. [17, Chapter II, §5, Theorem 5.16]). Moreover, by Corollary 2.5, $\left(T_{i}^{*}\right)$ is a BL-filtration on $E^{*}$. Consequently, by (c) and [14, Theorem 6.1], we have

$$
\begin{aligned}
& \mathcal{M}\left(E \widetilde{\otimes}_{l} Y, T_{i} \otimes_{l} \operatorname{id}_{Y}\right)=\mathcal{M}\left(E^{* *} \widetilde{\otimes}_{l} Y, T_{i}^{* *} \otimes_{l} \operatorname{id}_{Y}\right) \\
& \quad=\mathcal{M}_{\mathrm{f}}\left(E^{* *} \widetilde{\otimes}_{l} Y, T_{i}^{* *} \otimes_{l} \operatorname{id}_{Y}\right)=\mathcal{M}_{\mathrm{f}}\left(E \widetilde{\otimes}_{l} Y, T_{i} \otimes_{l} \operatorname{id}_{Y}\right) .
\end{aligned}
$$

Since the BL-filtration $\left(T_{i}\right)$ is complemented by a (positive) contractive projection $T_{\infty}: E \rightarrow E$, we have by [2, Lemma 4.2 and Lemma 5.1] that $\mathcal{R}\left(T_{\infty} \otimes_{l} \operatorname{id}_{Y}\right)=$ $\mathcal{R}\left(T_{\infty}\right) \widetilde{\otimes}_{l} Y=\overline{\bigcup_{i=1}^{\infty} \mathcal{R}\left(T_{i}\right)} \widetilde{\otimes}_{l} Y=\overline{\bigcup_{i=1}^{\infty} \mathcal{R}\left(T_{i} \otimes_{l} \text { id }_{Y}\right)}$. It now follows by a continuity argument that $\left(T_{i} \otimes_{l} \mathrm{id}_{Y}\right)$ is a filtration on $E \widetilde{\otimes}_{l} Y$ complemented by $T_{\infty} \otimes_{l} \mathrm{id}_{Y}$. Thus, $\mathcal{M}\left(E \widetilde{\otimes}_{l} Y, T_{i} \otimes_{l} \operatorname{id}_{Y}\right)=\mathcal{M}_{\mathrm{nc}}\left(E \widetilde{\otimes}_{l} Y, T_{i} \otimes_{l} \mathrm{id}_{Y}\right)$, as required.

(d) $\Rightarrow$ (a) For all finite measure spaces $(\Omega, \Sigma, \mu)$ and $1<p<\infty$, the Banach lattice $L^{p}(\mu)$ is separable and reflexive. By $(\mathrm{d})$, it follows that $\mathcal{M}\left(L^{p}(\mu, Y), \Sigma_{i}\right)=$ $\mathcal{M}_{\text {nc }}\left(L^{p}(\mu, Y), \Sigma_{i}\right)$ for every filtration $\left(\Sigma_{i}\right)$. Thus, $Y$ has the Radon Nikodým property by [9, Theorem II.2.2.2].

$(\mathrm{d}) \Leftrightarrow(\mathrm{e}) \quad$ Suppose $\mathcal{M}\left(E \widetilde{\otimes}_{l} Y, T_{i} \otimes_{l} \operatorname{id}_{Y}\right)=\mathcal{M}_{\mathrm{nc}}\left(E \widetilde{\otimes}_{l} Y, T_{i} \otimes_{l} \mathrm{id}_{Y}\right)$. Since $\left(T_{i}\right)$ is a BL-filtration, it follows that $\mathcal{M}\left(E \widetilde{\otimes}_{l} Y, T_{i} \otimes_{l}\right.$ id $\left._{Y}\right)=\mathcal{M}_{\mathrm{nc}}\left(E, T_{i}\right) \widetilde{\otimes}_{l} Y$ by [2, Corollary 5.2]. Since $E$ is reflexive, $\mathcal{M}_{\mathrm{nc}}\left(E, T_{i}\right)$ is Riesz and isometrically isomorphic to $\mathcal{M}\left(E, T_{i}\right)$. Thus, $\mathcal{M}\left(E \widetilde{\otimes}_{l} Y, T_{i} \otimes_{l}\right.$ id $\left._{Y}\right)=\mathcal{M}\left(E, T_{i}\right) \widetilde{\otimes}_{l} Y$ by 14, Theorem 6.1]. Conversely, using [2, Corollary 5.2] and [14, Theorem 6.1] again, we obtain $\mathcal{M}\left(E \widetilde{\otimes}_{l} Y, T_{i} \otimes_{l} \operatorname{id}_{Y}\right)=\mathcal{M}\left(E, T_{i}\right) \widetilde{\otimes}_{l} Y=\mathcal{M}_{\mathrm{nc}}\left(E, T_{i}\right) \widetilde{\otimes}_{l} Y=\mathcal{M}_{\mathrm{nc}}\left(E \widetilde{\otimes}_{l} Y, T_{i} \otimes_{l} \operatorname{id}_{Y}\right)$, as required. 
The above theorem allows us to generalize [5, Chapter IV, $\S 1$, Theorem 1], which characterizes the "Asplund spaces". A Banach space $Y$ is called an Asplund space if $Y^{*}$ has the Radon Nikodým property.

Corollary 3.9. Let $Y$ be a Banach space. Then $Y$ is an Asplund space if and only if $E^{*} \widetilde{\otimes}_{l} Y^{*}=\left(E \widetilde{\otimes}_{l} Y\right)^{*}$ for all separable Banach lattices $E$ with order continuous dual.

Proof. By Theorem 3.8, $Y^{*}$ has the Radon Nikodým property if and only if $E^{*} \widetilde{\otimes}_{l} Y^{*}$ $=\mathcal{L}^{\text {cas }}\left(E, Y^{*}\right)$ for all separable Banach lattices $E$ with order continuous dual. But, by a theorem of Jacobs, we have $\mathcal{L}^{\text {cas }}\left(E, Y^{*}\right)=\left(E \widetilde{\otimes}_{l} Y\right)^{*}$ (cf. [10, 17, Chapter IV, $\S 7$, Theorem 7.4] and [3]). Thus, $E^{*} \widetilde{\otimes}_{l} Y^{*}=\mathcal{L}^{\operatorname{cas}}\left(E, Y^{*}\right)=\left(E \widetilde{\otimes}_{l} Y\right)^{*}$, which completes the proof.

It is important to note that the above theorem does not include the case $E=$ $L^{1}(\mu)$. However, by [5, Chapter IV, $\S 1$, Theorem 1], $Y$ is an Asplund space if and only if $L^{1}(\mu, Y)^{*}=L^{\infty}\left(\mu, Y^{*}\right)$ for all finite measure spaces $(\Omega, \Sigma, \mu)$.

Combining Theorem 3.8 with [2, Theorem 5.3] yields another corollary:

Corollary 3.10. Let $Y$ be a Banach space. Then the following conditions are equivalent:

(a) $Y$ has the Radon Nikodým property.

(b) For every separable reflexive Banach lattice $E$ and every complemented, quasi-interior preserving BL-filtration $\left(T_{i}\right)$ on $E$, we have $\left(f_{n}\right) \in$ $\mathcal{M}\left(E \widetilde{\otimes}_{l} Y, T_{i} \otimes_{l} \mathrm{id}_{Y}\right)$ if and only if for each $i \in \mathbb{N}$, there exist $\left(x_{i}^{(n)}, T_{n}\right)_{n=1}^{\infty}$ $\in \mathcal{M}_{\mathrm{nc}}\left(E, T_{i}\right)$ and $y_{i} \in Y$ such that, for each $n \in \mathbb{N}$, we have $f_{n}=$ $\sum_{i=1}^{\infty} x_{i}^{(n)} \otimes y_{i}$, where $\left\|\sum_{i=1}^{\infty}\left|\lim _{n \rightarrow \infty} x_{i}^{(n)}\right|\right\|<\infty$ and $\lim _{i \rightarrow \infty}\left\|y_{i}\right\|=0$.

\section{ACKNOWLEDGEMENT}

The authors wish to express their gratitude to the referee, whose careful consideration exposed some gaps in our reasoning.

\section{REFERENCES}

1. J. Chaney, Banach lattices of compact maps, Math. Z. 129 (1972), 1-19. MR0312329(47:891)

2. S.F. Cullender and C.C.A. Labuschagne, A description of norm-convergent martingales on vector-valued $L^{p}$-spaces, J. Math. Anal. Appl. 323 (2006), 119-130. MR2261155|(2007j:60067)

3. A note on the M-norm of Chaney-Schaefer, Quaestiones Math. 30 (2007), no. 2, 151-158. MR2337359

4. A. Defant and K. Floret, Tensor norms and operator ideals, Mathematics Studies, no. 176, North-Holland, Amsterdam, London, New York, Tokyo, 1993. MR.1209438 (94e:46130)

5. J. Diestel and J.J. Uhl, Vector measures, Mathematical Surveys, no. 15, A.M.S., Providence, Rhode Island, 1977. MR0453964 (56:12216)

6. N. Dinculeanu, Integral representation of linear operators. I, Stud. Cerc. Mat. 18 (1966), 349-385. MR0213504 (35:4366a)

7. Integral representation of linear operators II, Stud. Cerc. Mat. 18 (1966), 483-536. MR:0213505 (35:4366b)

8. Linear operations on $L^{p}$-spaces, Vector and Operator Valued Measures and Applications, Academic Press, New York, 1973, pp. 109-124. MR0341066 (49:5816)

9. L. Egghe, Stopping time techniques for analysts and probabilists, London Mathematical Society Lecture Note Series, no. 100, Cambridge University Press, London, New York, New Rochelle, Melbourne, Sydney, 1984. MR808582 (87j:60070)

10. H. Jacobs, Ordered topological tensor products, Ph.D. thesis, University of Illinois, 1969. 
11. G.A.M. Jeurnink, Integration of functions with values in a Banach lattice, Ph.D. thesis, University of Nijmegen, The Netherlands, 1982.

12. S. Kakutani, Concrete representation of abstract $L$-spaces and the mean ergodic theorem, Ann. of Math. 42 (1941), 523-537. MR.0004095 (2:318d)

13. J.L. Krivine, Théorèmes de factorisation dans les espaces réticulés, Séminaire MaureySchwartz 1973-1974 (École Polytechnique, Paris), Exposés 22-23, 1974. MR0440334 (55:13209)

14. C.C.A. Labuschagne, Characterizing the one-sided tensor norms $\Delta_{p}$ and ${ }^{t} \Delta_{p}$, Quaestiones Math. 27 (2004), 339-363. MR2125036 (2005m:46036)

15. J. Lindenstrauss and L. Tzafriri, Classical Banach spaces II, A Series of Modern Surveys in Mathematics, no. 97, Springer-Verlag, Berlin, Heidelberg, New York, 1979. MR540367 (81c:46001)

16. P. Meyer-Nieberg, Banach lattices, Springer-Verlag, Heidelberg, Berlin, New York, 1991. MR.1128093 (93f:46025)

17. H.H. Schaefer, Banach lattices and positive operators, Die Grundlehren der Mathematischen Wissenschaften, no. 215, Springer-Verlag, Berlin, Heidelberg, New York, 1974. MR 0423039 (54:11023)

18. V. Troitsky, Martingales in Banach lattices, Positivity 9 (2005), 437-456. MR2188530 (2006j:60045)

School of Computational and Applied Mathematics, University of the WitwaterSRand, Private Bag 3, P.O. Wits 2050, South Africa

E-mail address: scullender@gmail.com

School of Computational and Applied Mathematics, University of the WitwaterSRand, Private Bag 3, P.O. Wits 2050, South Africa

E-mail address: Coenraad.Labuschagne@wits.ac.za 